TITLE:

\title{
Moment Restriction-Based Econometric Methods: An overview
}

\section{$\operatorname{AUTHOR}(S)$ :}

Kunitomo, Naoto; McAleer, Michael; Nishiyama, Yoshihiko

\section{CITATION:}

Kunitomo, Naoto ... [et al]. Moment Restriction-Based Econometric Methods: An overview. Journal of Econometrics 2011, 165(1): 1-4

ISSUE DATE:

2011-11

URL:

http://hdl.handle.net/2433/148010

\section{RIGHT:}

C 2011 Elsevier B.V.; この論文は出版社版でありません。引用の際には 出版社版をご確認ご利用ください。; This is not the published version. Please cite only the published version. 


\title{
Moment Restriction-based Econometric Methods: An Overview
}

\author{
Naoto Kunitomo \\ Faculty of Economics \\ University of Tokyo \\ Japan
}

Michael McAleer

Econometric Institute

Erasmus School of Economics

Erasmus University Rotterdam

and

Tinbergen Institute

The Netherlands

and

Institute of Economic Research

Kyoto University

Japan

Yoshihiko Nishiyama

Institute of Economic Research

Kyoto University

Japan

October 2006 


\begin{abstract}
Moment restriction-based econometric modelling is a broad class which includes the parametric, semiparametric and nonparametric approaches. Moments and conditional moments themselves are nonparametric quantities. If a model is specified in part up to some finite dimensional parameters, this will provide semiparametric estimates or tests. If we use the score to construct moment restrictions to estimate finite dimensional parameters, this yields maximum likelihood (ML) estimates. Semiparametric or nonparametric settings based on moment restrictions have been the main concern in the literature, and comprise the most important and interesting topics. The purpose of this special issue on "Moment Restriction-based Econometric Methods" is to highlight some areas in which novel econometric methods have contributed significantly to the analysis of moment restrictions, specifically asymptotic theory for nonparametric regression with spatial data, a control variate method for stationary processes, method of moments estimation and identifiability of semiparametric nonlinear errors-in-variables models, properties of the CUE estimator and a modification with moments, finite sample properties of alternative estimators of coefficients in a structural equation with many instruments, instrumental variable estimation in the presence of many moment conditions, estimation of conditional moment restrictions without assuming parameter identifiability in the implied unconditional moments, moment-based estimation of smooth transition regression models with endogenous variables, a consistent nonparametric test for nonlinear causality, and linear programming-based estimators in simple linear regression.
\end{abstract}

Keywords: Moment restrictions, Parametric, semiparametric and nonparametric methods; Estimation; Testing; Robustness; Model misspecification. 


\section{Introduction}

One of the primary missions of econometric theory is to provide statistical methods to estimate unknown key quantities and to test hypotheses relating to these quantities. The optimal statistical strategy for estimation and testing is based on the likelihood principle, given a sufficiently large number of observations. In order for this implementation, it is necessary to have the joint (or conditional) distribution of the economic variables. Economic theory, however, is typically unable to supply a full model which completely specifies the statistical structure, namely a parametric statistical model. In empirical studies, it is common to use the maximum likelihood method to estimate unknown parameters under the assumption of, say, normality of the disturbances, such as in regression analysis and limited dependent variable models, among others.

If the specified model is correct, it is possible to obtain optimal results asymptotically. However, there is a severe limitation in that the estimates or test statistics may be unreliable if the assumed parametric model is, in fact, incorrect. In short, there exists a trade-off between efficiency (under the null hypothesis of correct specification) and robustness (under the alternative of incorrect specification). In view of this possibility, it may be more sensible to assume a less restrictive model or structure which will yield more robust statistical outcomes.

A viable alternative to the parametric approach is to assume only certain conditional or unconditional moment conditions without specifying a fully parametric model. Indeed, it has become common practice to use partially specified econometric models that are described in terms of a variety of moment restrictions. Econometric theory has contributed significantly to modelling using moment restrictions over the last half century.

Formally, moment restriction-based econometric modelling is a broad class which includes the parametric, semiparametric and nonparametric approaches. To state the obvious, moments and conditional moments themselves are nonparametric quantities, so that if we estimate or test certain unconditional or conditional moments without 
further restrictions, it must be a nonparametric procedure. If the model is specified in part up to some finite dimensional parameters, this will provide semiparametric estimates or tests. Examples include partially linear regression models and index models. If we use the score to construct moment restrictions to estimate finite dimensional parameters, this yields maximum likelihood (ML) estimates. It would seem that semiparametric or nonparametric settings based on moment restrictions have been the main concern in the literature and, in our view, comprise the most important and interesting topics in this context. Some of the basic advantages and disadvantages of the parametric, semiparametric and nonparametric approaches are given in Table 1.

\section{[Table 1 goes here]}

Parametric modelling performs best if the specified model is correctly specified, but the optimal properties generally disappear under misspecification. Nonparametric modelling is hardly incorrect as it typically does not assume any structure other than some smoothness conditions, and hence does not face the consequences of possible misspecification. However, nonparametric estimates are sometimes difficult to interpret, for example, in the case of nonparametric multiple regression. However, nonparametric methods can provide a very useful tool for testing, as in specification tests, where it is possible to obtain reliable results in comparing parametric versus nonparametric models or semparametric versus nonparametric models. Semiparametric modelling is a good compromise in estimation between parametric and nonparametric in most cases as it balances efficiency versus robustness. Consequently, moment restriction-based econometric methods can easily fit into either the nonparametric or semiparametric framework.

The instrumental variables (IV) method is perhaps the most basic and useful tool in econometric estimation based on moment restrictions. IV is used when least squares (LS) estimation is inappropriate due to endogeneity in models such as simultaneous equation systems, regressions with errors in variables, and autoregressive models with serially correlated disturbances, among others. When we have more instruments than are required for identification, we can use the generalized method of moments (GMM) estimation method, as proposed by Hansen (1982). GMM is undoubtedly one 
of the most widely used methods in contemporary econometrics, and is typically a semiparametric estimator, where the moment condition is described in terms of the observations, a finite number of parameters and function(s) relating these quantities. It is widely known in the statistics literature that Godambe (1960) also proposed what is essentially an equivalent method, namely the estimating equation method, which bridges ML estimation and the method of moments estimation. Estimation methods that have the same first-order asymptotic properties as GMM were proposed in the 1980s and 1990s, such as the empirical likelihood and exponential tilting estimators, and further a general class called generalized empirical likelihood estimator, which includes all of the above as special cases. We also would like to mention an important class of econometric analysis called entropy based econometric methods, which takes an information theoretic approach but is closely related to the present theme, as well as maximum likelihood approach. We refer to Golan (2002) which appeared in another special volume of the Journal of Econometrics, and the articles therein.

Specification analysis is a classical and important issue in econometrics. A classical example is the t-test in linear regression, where we are interested in whether a certain independent variable is useful in accounting for the variation in the dependent variable. Various extensions to nonparametric and semiparametric settings have been considered in the literature since the 1980s by Bierens (1980), among others. This is a specification test for conditional moments, where both the null and alternative hypotheses are nonparametric. These tests are called "omitted variable" tests rather than nonparametric significance tests. Härdle and Mammen (1993), for example, propose a specification test of a parametric regression versus a nonparametric regression. Semiparametric modelling partially specifies the structure, where it is often an issue whether the specified component can be justified on the basis of the data. For example, whether the regression function has a single index structure or not is something which should be checked in semiparametric single index models. Such tests of semiparametric models versus nonparametric counterpart have also been developed, for example, in Whang and Andrews (1993) and Yatchew (1992), among many others.

Many moments and weak instrument problems are important empirical issues in GMM estimation and its variants, which have been considered since the late 1990s. 
When there are many moment conditions, the bias tends to become large. Such a phenomenon has been known in the context of estimation for simultaneous equation systems since the 1980s. Kunitomo (1980) examined the asymptotic properties of the two-stage least squares estimator under many instruments, while Morimune (1983) provided the higher-order approximations of the distribution of the limited information maximum likelihood estimator under the same conditions. Weak instruments are instrumental variables which have very low correlations with the explanatory variables or endogenous variables. In such a case, the resulting estimate becomes very unstable, such that a small change in the variables leads to a large change in the estimates. The many moments problem and weak instruments problem tend to occur simultaneously because it is more likely that some instruments are weak when there are many instruments.

It is worth mentioning some directions in moment restriction-based econometrics in which econometricians are presently interested. In financial econometrics and empirical finance, forecasting of Value-at-Risk ( $\mathrm{VaR})$ and the corresponding quantiles is of increasing importance in developing optimal risk management strategies. Another important area is the analysis of treatment effects or program evaluation, in which the quantities of interest are identified with respect to moments or conditional moments. Various methods of identification and estimation have been developed during the last two decades, and have been usefully applied. A further important direction of recent advances is set estimation by moment inequalities. This kind of approach is mainly used in the econometric analysis of games because economic agents facing game theoretic situations typically select their actions by comparing their outcomes from a variety of strategies. Further theoretical developments and implementation will likely shed light on the behaviour of economic agents.

The purpose of this special issue on "Moment Restriction-based Econometric Methods" is to highlight some areas in which novel econometric methods have contributed significantly to the analysis of moment restrictions, specifically asymptotic theory for nonparametric regression with spatial data (Robinson, 2010), a control variate method for stationary processes (Amano and Taniguchi, 2010), method of moments estimation and identifiability of semiparametric nonlinear errors-invariables models (Wang and Hsiao, 2010), properties of the CUE estimator and a 
modification with moments (Hausman et al., 2010), finite sample properties of alternative estimators of coefficients in a structural equation with many instruments (Anderson et al., 2010), instrumental variable estimation in the presence of many moment conditions (Okui, 2010), estimation of conditional moment restrictions without assuming parameter identifiability in the implied unconditional moments (Hsu and Kuan, 2010), moment-based estimation of smooth transition regression models with endogenous variables (Areosa et al., 2010), a consistent nonparametric test for nonlinear causality (Nishiyama et al., 2010), and linear programming-based estimators in simple linear regression (Preve and Medeiros, 2010).

It is our hope that these invaluable papers, most of which were presented at a conference at Kyoto University in honour of Professor Kimio Morimune's 60'th birthday, will encourage others to undertake research in a variety of challenging areas associated with moment restriction-based econometric methods. Two excellent papers (among many) by Kimio Morimune are related to the main theme of the special issue. Although it was known that maximum likelihood estimation often led to an appropriate answer in parametric models, standard asymptotic theory often gave several asymptotically (equivalent) efficient methods. Takeuchi and Morimune (1985) gave a definitive classical answer to this problem in the context of a parametric estimation problem of structural equations, which corresponds to the estimating equation problem and the reduced rank regression problem. Morimune (1989) also gave a definitive answer to the statistical question of the standard use of $t$ tests in the structural equation problem, which is an impressive result.

\section{Overview}

In the first paper, Peter Robinson (London School of Economics) considers "Asymptotic theory for nonparametric regression with spatial data", specifically, nonparametric regression with spatial, or spatio-temporal, data. The conditional mean of a dependent variable, given explanatory variables, is a nonparametric function, while the conditional covariance reflects spatial correlation. Conditional heteroscedasticity is also allowed, as well as non-identically distributed observations. Instead of mixing conditions, a (possibly non-stationary) linear process is assumed for 
disturbances, allowing for long range, as well as short-range, dependence, while decay independence in explanatory variables is described using a measure based on the departure of the joint density from the product of marginal densities. A basic triangular array setting is employed, with the aim of covering various patterns of spatial observation. Sufficient conditions are established for consistency and asymptotic normality of kernel regression estimates. When the cross-sectional dependence is sufficiently mild, the asymptotic variance in the central limit theorem is the same as when observations are independent; otherwise the rate of convergence is slower. The author discusses application of the established conditions to spatial autoregressive models, and models defined on a regular lattice.

Continuing the theme of moment restriction-based econometric methods, Tomoyuki Amano (Waseda University) and Masanobu Taniguchi (Waseda University) develop a "Control variate method for stationary processes". The sample mean is a natural estimator of the population mean based on independently and identically distributed observations. However, if some control variate were available, it is known that the control variate method reduces the variance of the sample mean. The control variate method often assumes that the variable of interest and the control variable are independently and identically distributed. The authors assume that these variables are stationary processes with spectral density matrices, that is, they are dependent, then propose an estimator of the mean of the stationary process of interest by using a control variate method based on a nonparametric spectral estimator. It is shown that this estimator improves the sample mean in the sense of mean square error. The authors also extend the analysis to the case where the mean dynamics is of the regression form, and then propose a control variate estimator for the regression coefficients which improves the least squares estimator (LSE). Numerical studies show how their estimator improves the LSE.

Liqun Wang (University of Manitoba) and Cheng Hsiao (University of Southern California and Xiamen University) consider "Method of moments estimation and identifiability of semiparametric nonlinear errors-in-variables models". Their paper deals with a nonlinear errors-in-variables model, where the distributions of the unobserved predictor variables and of the measurement errors are nonparametric. Using the instrumental variable approach, the authors propose method of moments 
estimators for the unknown parameters and simulation-based estimators to overcome the possible computational difficulty of minimizing an objective function which involves multiple integrals. Both estimators are consistent and asymptotically normally distributed under fairly general regularity conditions. Moreover, root-n consistent semiparametric estimators and a rank condition for model identifiability are derived using the combined methods of nonparametric technique and Fourier deconvolution.

In the fourth paper, Jerry Hausman (MIT), Konrad Menzel (???), Randall Lewis (???) and Whitney Newey (MIT) analyse "Properties of the CUE estimator and a modification with moments". Specifically, the authors analyse the properties of the Continuous Updating Estimator (CUE) proposed by Hansen, Heaton and Yaron (1996), which has been suggested as a solution to the finite sample bias problems of the two-step GMM estimator. The authors show that the estimator has high dispersion infinite samples under weak identification, and propose the Regularized CUE (RCUE) as a solution to this problem. The RCUE solves a modification of the first-order conditions for the CUE estimator. The authors show that the RCUE is asymptotically equivalent to the CUE under many weak moment asymptotics, as in Newey and Windmeijer (2008). They provide extensive Monte Carlo results showing the low bias and dispersion properties of the new estimator.

"On finite sample properties of alternative estimators of coefficients in a structural equation with many instruments" leads T.W. Anderson (Stanford University), Naoto Kunitomo (University of Tokyo) and Yukitoshi Matsushita (University of Tokyo) to compare four different estimation methods for the coefficients of a linear structural equation with instrumental variables. As the classical methods, the authors consider the limited information maximum likelihood (LIML) estimator and the two-stage least squares (TSLS) estimator, and as the semi-parametric estimation methods, they consider the maximum empirical likelihood (MEL) estimator and the generalized method of moments (GMM) (or the estimating equation) estimator. Tables and figures of the distribution functions of four estimators are given for sufficient values of the parameters to cover most linear models of interest, and the authors include some heteroscedastic cases and nonlinear cases. The authors find that the LIML estimator has good performance in terms of the bounded loss functions and probabilities when 
the number of instruments is large, that is, for micro-econometric models with "many instruments", in the terminology of recent econometric literature.

In the sixth paper, "Instrumental variable estimation in the presence of many moment conditions", Ryo Okui (Hong Kong University of Science and Technology) develops shrinkage methods for addressing the "many instruments" problem in the context of instrumental variable estimation. It has been observed that instrumental variable estimators may behave poorly if the number of instruments is large. This problem can be addressed by shrinking the influence of a subset of instrumental variables. The procedure can be understood as a two-step process of shrinking some of the OLS coefficient estimates from the regression of the endogenous variables on the instruments, then using the predicted values of the endogenous variables (based on the shrunk coefficient estimates) as the instruments. The shrinkage parameter is chosen to minimize the asymptotic mean square error. The optimal shrinkage parameter has a closed form, which makes it easy to implement. A Monte Carlo study shows that the shrinkage method works well and performs better in many situations than do existing instrument selection procedures.

Shih-Hsun Hsu (National Chengchi University) and Chung-Ming Kuan ( National Taiwan University) examine "Estimation of conditional moment restrictions without assuming parameter identifiability in the implied unconditional moments". A well known difficulty in estimating conditional moment restrictions is that the parameters of interest need not be globally identified by the implied unconditional moments. The authors propose an approach to constructing a continuum of unconditional moments that can ensure parameter identifiability. These unconditional moments depend on the instruments "generated from a generically comprehensively revealing" function, and they are further projected along the exponential Fourier series. The objective function is based on the resulting Fourier coefficients, from which an estimator can be easily computed. A novel feature of the new method is that the full continuum of unconditional moments is incorporated into each Fourier coefficient. The authors show that, when the number of Fourier coefficients in the objective function grows at a proper rate, the proposed estimator is consistent and asymptotically normally distributed. An efficient estimator is also readily obtained via the conventional twostep GMM method. The simulations confirm that the proposed estimator compares 
favourably with that of Dominguez and Lobato (2004) in terms of bias, standard error, and mean squared error.

In the eighth paper, Waldyr Dutra Areosa (Pontifical Catholic University of Rio de Janeiro), Michael McAleer (Erasmus University Rotterdam) and Marcelo C. Medeiros (Pontifical Catholic University of Rio de Janeiro) evaluate "Moment-based estimation of smooth transition regression models with endogenous variables". Nonlinear regression models have been widely used for time series and cross-section datasets. For analyzing univariate and multivariate time series data, in particular, Smooth Transition Regression (STR) models have been shown to be very useful for representing and capturing asymmetric behaviour. Most STR models have been applied to univariate processes, and have made a variety of assumptions, including stationary or cointegrated processes, uncorrelated, homoskedastic or conditionally heteroskedastic errors, and weakly exogenous regressors. Under the assumption of exogeneity, the standard method of estimation is nonlinear least squares. The authors relax the assumption of weakly exogenous regressors and discuss moment-based methods for estimating STR models. They analyze the properties of the STR model with endogenous variables with a diagnostic test of linearity under endogeneity, developing an estimation procedure and a misspecification test for the STR model, present results of Monte Carlo simulations to show the usefulness of the model and estimation method, and providing an empirical application for inflation rate targeting in Brazil. They show that STR models with endogenous variables can be specified and estimated by a straightforward application of existing results.

Yoshiohiko Nishiyama (Kyoto University), Kohtaro Hitomi (Kyoto Institute of Technology), Yoshinori Kawasaki (Institute of Statistical Mathematics) and Kiho Jeong (Kyunpook National University) develop “A consistent nonparametric test for nonlinear causality”. Following Granger (1969), many tests have been proposed for causality of economic time series. Most have been concerned only with "linear causality in mean". It is undoubtedly of primary interest, but dependence between series may be nonlinear, and/or not only through the conditional mean, such as conditional heteroskedasticity models. The authors propose a nonparametric test for possibly nonlinear causality. Taking into account that dependence in higher-order moments are established as an important issue, especially in financial time series, the 
authors also consider a test for causality up to the $K$-th conditional moment. Statistically, their new test can be considered as a nonparametric omitted variable test in time series regression. A desirable property of the test is that it has nontrivial power against square-root- $T$-local alternatives, where $T$ is the sample size. It is possible to develop a test statistic accordingly if there is some knowledge regarding the alternative hypothesis. Furthermore, the authors show that the test statistic includes most of the established omitted variables test statistics as special cases asymptotically. The null asymptotic distribution is not normal, but the critical regions can be calculated by simulation. Monte Carlo experiments show that the proposed test has good size and power properties.

In the final paper, Daniel Preve (Singapore Management University) and Marcelo C. Medeiros (Pontifical Catholic University of Rio de Janeiro) consider "Linear programming-based estimators in simple linear regression". The authors introduce a linear programming estimator (LPE) for the slope parameter in a constrained linear regression model with a single regressor. The LPE is interesting because it can be superconsistent in the presence of an endogenous regressor and, hence, preferable to the ordinary least squares estimator (LSE). One advantage of the LPE is that it does not require an instrument. Two different cases are considered as the authors investigate the statistical properties of the LPE. In the first case, the regressor is assumed to be fixed in repeated samples, and in the second, the regressor is stochastic and potentially endogenous. In both cases, the strong consistency and exact finitesample distribution of the LPE are established Conditions under which the LPE is consistent in the presence of serially correlated and heteroskedastic errors are also given. Finally, the authors describe how the LPE can be extended to the case with multiple regressors, and conjecture that the extended estimator is consistent under conditions analogous to those given in the paper. Finite-sample properties of the LPE and extended LPE in comparison to the LSE and instrumental variable estimator (IVE) are investigated in a simulation study.

It is hoped that this collection of papers by some of the leading experts in the field of "Moment Restriction-based Econometric Methods" will be of wide interest to a diverse range of econometricians, statisticians, and quantitative researchers. It is a genuine pleasure to acknowledge all of the contributors for preparing their innovative 
submissions in a timely manner, and for actively participating, together with others, in the rigorous review process.

\section{Acknowledgements}

The authors wish to thank numerous referees for their helpful, insightful and timely reviews of the papers that comprise this special issue. The second author wishes to acknowledge the financial support of the Australian Research Council, National Science Council, Taiwan, and the Japan Society for the Promotion of Science. 


\section{References}

Amano, T. and M. Taniguchi (2010), Control variate method for stationary processes, to appear in Journal of Econometrics, this issue.

Anderson, T.W., N. Kunitomo and Y. Matsushita (2010), On finite sample properties of alternative estimators of coefficients in a structural equation with many instruments, to appear in Journal of Econometrics, this issue.

Areosa, W.D., M. McAleer and M.C. Medeiros (2010), Moment-based estimation of smooth transition regression models with endogenous variables, to appear in Journal of Econometrics, this issue.

Bierens, H.J. (1990), A consistent conditional moment test of functional form, Econometrica, 58(6), 1443-1458.

Fan, Y. and Q. Li (1996), Consistent model specification tests: Omitted variables and semiparametric functional forms, Econometrica, 64(4), 865-890.

Godambe, V.P. (1960), An optimum property of regular maximum likelihood estimation, Annals of Mathematical Statistics 31, 1208-1211.

Golan, A. (2002), Information and Entropy Econometrics-Editor's view, Journal of Econometrics, 107, 1, 1-15.

Hansen, L.P. (1982), Large sample properties of generalized method of moments estimators, Econometrica, 50(4), 1029-1054.

Härdle, W. and E. Mammen (1993), Comparing nonparametric versus parametric regression fits, Annals of Statistics, 21, 1926-1947.

Hausman, J., K. Menzel, R. Lewis and W. Newey (2010), Properties of the CUE estimator and a modification with moments, to appear in Journal of Econometrics, this issue.

Hsu, S.-H. and C.-M. Kuan (2010), Estimation of conditional moment restrictions without assuming parameter identifiability in the implied unconditional moments, to appear in Journal of Econometrics, this issue.

Kunitomo, N. (1980), Asymptotic expansions of the distributions of estimators in a linear functional relationship and simultaneous equations, Journal of the American Statistical Association, 75, 693-700.

Morimune, K. (1983), Approximate distributions of k-class estimators when the degree of overidentification is large compared with the sample size, Econometrica, 51, 821-841.

Morimune, K. (1989), t tests in a structural equation, Econometrica, 57, 1341-1360. 
Nishiyama, Y., K. Hitomi, Y. Kawasaki and K. Jeong (2010), A consistent nonparametric test for nonlinear causality, to appear in Journal of Econometrics, this issue.

Okui, R. (2010), Instrumental variable estimation in the presence of many moment conditions, to appear in Journal of Econometrics, this issue.

Preve, D. and M.C. Medeiros (2010), Linear programming-based estimators in simple linear regression, to appear in Journal of Econometrics, this issue.

Robinson, P.M. (2010), Asymptotic theory for nonparametric regression with spatial data, to appear in Journal of Econometrics, this issue.

Takeuchi, K. and K. Morimune (1985), Third order efficiency of the extended maximum likelihood estimator in a linear simultaneous equation system, Econometrica, 53, 177-200.

Wang, L. and C. Hsiao (2010), Method of moments estimation and identifiability of semiparametric nonlinear errors-in-variables models, to appear in Journal of Econometrics, this issue.

Whang, Y. and D.W.K. Andrews (1993), Tests of specification for parametric and semiparametric models, Journal of Econometrics, 57, 277-318.

Yatchew, A.J. (1992), Nonparametric regression tests based on least squares, Econometric Theory, 8, 435-451. 
Table 1

Advantages and Disadvantages of Parametric, Semiparametric and Nonparametric Methods

\begin{tabular}{|l|l|l|}
\hline \multicolumn{1}{|c|}{ Method } & \multicolumn{1}{|c|}{ Advantages } & \multicolumn{1}{c|}{ Disadvantages } \\
\hline Parametric & $\begin{array}{l}\text { (i) Efficient estimation; } \\
\text { (ii) Sample size can be small; } \\
\text { (iii) Powerful tests against } \\
\text { parametric alternatives. }\end{array}$ & $\begin{array}{l}\text { (i) Estimation inconsistency under } \\
\text { model misspecification; } \\
\text { (ii) Test inconsistency under } \\
\text { incorrect parametric alternatives. }\end{array}$ \\
\hline $\begin{array}{l}\text { Semiparametr } \\
\text { ic }\end{array}$ & $\begin{array}{l}\text { Tries to balance efficiency versus } \\
\text { robustness. }\end{array}$ & $\begin{array}{l}\text { Inconsistency under model } \\
\text { misspecification. }\end{array}$ \\
\hline Nonparametri & $\begin{array}{l}\text { (i) Robustness; } \\
\text { (ii) Can have powerful tests even } \\
\text { if the alternative is incorrect. }\end{array}$ & $\begin{array}{l}\text { (i) Inefficient estimation; } \\
\text { (ii) Requires large samples; } \\
\text { (iii) Can be difficult to interpret } \\
\text { estimates. }\end{array}$ \\
\hline
\end{tabular}

